International Journal of Pure and Applied Mathematics

Volume 88 No. 4 2013, 499-522

ISSN: 1311-8080 (printed version); ISSN: 1314-3395 (on-line version)

url: http://www.ijpam.eu

doi: http://dx.doi.org/10.12732/ijpam.v88i4.5

\title{
ON THE EXISTENCE OF SOLUTIONS OF THE FIRST BOUNDARY VALUE PROBLEM FOR ELLIPTIC EQUATIONS ON UNBOUNDED DOMAINS
}

\author{
Armen L. Beklaryan \\ Department of Business Analytics \\ Higher School of Economics \\ National Research University \\ 33, Kirpichnaya Str., Moscow, 105187, RUSSIA
}

Abstract: In this paper we consider the first boundary value problem for elliptic systems, defined on unbounded domains $\Omega \subset \mathbb{R}^{n}$, which solutions satisfy a condition of finiteness of the Dirichlet integral, also known as the energy integral

$$
\int_{\Omega}|\nabla u|^{2} d x<\infty
$$

AMS Subject Classification: $35 \mathrm{~J} 25$

Key Words: elliptic equation, boundary value problem, Hardy inequality, capacity

\section{Introduction}

Let $\Omega$ be an unbounded open subset of $\mathbb{R}^{n}, n \geq 2$. As is customary, by $W_{2, l o c}^{1}(\Omega)$ we denote the space of functions which are locally Sobolev, i.e.,

$$
W_{2, l o c}^{1}(\Omega)=\left\{f: f \in W_{2}^{1}\left(\Omega \cap B_{\rho}^{x}\right) \forall \rho>0, \forall x \in \mathbb{R}^{n}\right\},
$$

where $B_{\rho}^{x}$ the open ball in $\mathbb{R}^{n}$ of radius $\rho$ centered at the point $x$ [9]. If $x=0$, we write $B_{\rho}$ instead of $B_{\rho}^{x}$. In this case, denote by $\stackrel{\circ}{W}_{2}^{1}$ loc $(\Omega)$ the subset of $W_{2, \text { loc }}^{1}\left(\mathbb{R}^{n}\right)$ which is the closure of $C_{0}^{\infty}(\Omega)$ in the system of seminorms

Received: June 10, 2013

(C) 2013 Academic Publications, Ltd. url: www.acadpubl.eu 
$\|u\|_{W_{2}^{1}\left(\Omega \cap B_{\rho}\right)}, \rho>0$. Further, following [10, Subsec. 1.1], denote by $L_{2}^{1}(\Omega)$ the space of distributions ("generalized functions") whose first derivatives belong to $L_{2}(\Omega)$; in other words,

$$
L_{2}^{1}(\Omega)=\left\{f \in \mathcal{D}^{\prime}(\Omega): \int_{\Omega}|\nabla f|^{2} d x<\infty\right\} .
$$

Let $\omega \subseteq \mathbb{R}^{n}$ be an open set and let $\mathcal{K} \subset \omega$ be a compact set. Denote by $\Phi_{\varphi}(\mathcal{K}, \omega)$ the set of functions $\psi \in C_{0}^{\infty}(\omega)$ such that $\psi=\varphi$ in a neighborhood of $\mathcal{K}$, or, in other words, $\psi-\varphi \in \stackrel{\circ}{W}_{2, \text { loc }}^{1}\left(\mathbb{R}^{n} \backslash \mathcal{K}\right)$. Write $\Psi(\mathcal{K}, \omega)=\{\psi \in$ $C_{0}^{\infty}(\omega): \psi=1$ in a neighborhood of $\mathcal{K}$.

The quantity

$$
\operatorname{cap}_{\varphi}(\mathcal{K}, \omega)=\inf _{\psi \in \Phi_{\varphi}(\mathcal{K}, \omega)} \int_{\omega}|\nabla \psi|^{2} d x
$$

is referred to as the capacity of the compact set $\mathcal{K}$ with respect to an open set $\omega\left[10\right.$, Subsec. 7.2]. The capacity of an arbitrary closed subset $E \subset \omega$ of $\mathbb{R}^{n}$ is defined by the rule

$$
\operatorname{cap}_{\varphi}(E, \omega)=\sup _{\mathcal{K} \subset E} \operatorname{cap}_{\varphi}(\mathcal{K}, \omega)
$$

where the supremum on the right-hand side is taken over all compacta $\mathcal{K} \subset E$. If $\omega=\mathbb{R}^{n}$, then we write $\operatorname{cap}_{\varphi}(E)$ instead of $\operatorname{cap}_{\varphi}\left(E, \mathbb{R}^{n}\right)$.

We also need the following capacity [10, Subsec. 9.1]:

$$
\operatorname{Cap}\left(\mathcal{K}, W_{2}^{1}(\omega)\right)=\inf _{\psi \in \Psi(\mathcal{K}, \omega)}\left(\int_{\omega}|\nabla \psi|^{2} d x+\int_{\omega}|\psi|^{2} d x\right)
$$

As above, the capacity of an arbitrary set $E \subset \omega$ closed in $\mathbb{R}^{n}$ is given by the rule

$$
\operatorname{Cap}\left(E, W_{2}^{1}(\omega)\right)=\sup _{\mathcal{K} \subset E} \operatorname{Cap}\left(\mathcal{K}, W_{2}^{1}(\omega)\right)
$$

where the supremum on the right-hand side is taken over all compacta $\mathcal{K} \subset E$.

Finally, denote by $W_{2}^{-1}$ the space of continuous linear functionals on $W_{2}^{1}$. A set $E \subset \mathbb{R}^{n}$ is said to be (2,1)-polar if the only element of $W_{2}^{-1}$ supported by $E$ is zero [10, Subsec. 9.2]. 


\section{Statement of the Problem}

Here and below, $L$ stands for the divergence operator of the form

$$
L=\sum_{i, j=1}^{n} \frac{\partial}{\partial x_{i}}\left(a_{i j}(x) \frac{\partial}{\partial x_{j}}\right)
$$

where measurable bounded coefficients $a_{i j}$ satisfy the uniform ellipticity condition

$$
c_{1}|\xi|^{2} \leq \sum_{i, j=1}^{n} a_{i j}(x) \xi_{i} \xi_{j} \leq c_{2}|\xi|^{2}, \quad \xi \in \mathbb{R}^{n}, \quad c_{1}, c_{2}>0 .
$$

By a solution of the Dirichlet problem

$$
\left\{\begin{array}{l}
L u=0 \text { on } \Omega \\
\left.u\right|_{\partial \Omega}=\varphi
\end{array}\right.
$$

where $\varphi \in W_{2, l o c}^{1}\left(\mathbb{R}^{n}\right)$, we mean a function $u \in W_{2, l o c}^{1}(\Omega)$ such that

1) $u-\varphi \in \stackrel{\circ}{W}_{2, l o c}^{1}(\Omega)$, i.e., $(u-\varphi) \mu \in \stackrel{\circ}{W}_{2}^{1}(\Omega)$ for any function $\mu \in C_{0}^{\infty}\left(\mathbb{R}^{n}\right)$;

2) the function $u$ has the bounded Dirichlet integral

$$
\int_{\Omega}|\nabla u|^{2} d x<\infty
$$

3)

$$
\int_{\Omega} \sum_{i, j=1}^{n} a_{i j}(x) \frac{\partial u}{\partial x_{j}} \frac{\partial \psi}{\partial x_{i}} d x=0
$$

for any function $\psi \in C_{0}^{\infty}(\Omega)$.

\section{Main Results}

Theorem 1. Let $\operatorname{cap}_{\varphi-c}\left(\mathbb{R}^{n} \backslash \Omega\right)<\infty$ for some constant $c \in \mathbb{R}$. Then problem (1) has a solution.

Theorem 2. Let problem (1) have a solution, and let

$$
\int_{\mathbb{R}^{n} \backslash \Omega}|\nabla \varphi|^{2} d x<\infty .
$$

Then there is a constant $c \in \mathbb{R}$ such that $\operatorname{cap}_{\varphi-c}\left(\mathbb{R}^{n} \backslash \Omega\right)<\infty$. 
Theorem 3. For any function $\varphi \in W_{2, l o c}^{1}\left(\mathbb{R}^{n}\right)$, the condition $\operatorname{cap}_{\varphi-c}\left(\mathbb{R}^{n} \backslash\right.$ $\Omega)<\infty$ is equivalent to the inequality

$$
\sum_{k=1}^{\infty} \operatorname{cap}_{\varphi-c}\left(\left(\bar{B}_{r_{k+1}} \backslash B_{r_{k-1}}\right) \cap\left(\mathbb{R}^{n} \backslash \Omega\right), B_{r_{k+2}} \backslash \bar{B}_{r_{k-2}}\right)<\infty
$$

where

$$
r_{k}= \begin{cases}2^{k}, & \text { if } n \geq 3 \\ 2^{2^{k}}, & \text { if } n=2 .\end{cases}
$$

Let $\omega \subset \mathbb{R}^{n}$ be a bounded Lipschitz domain, and let $\mu$ be a measure on $\omega$ such that

$$
\sup _{x \in \mathbb{R}^{n}, \rho>0} \rho^{1-n} \mu\left(B_{\rho}^{x} \cap \omega\right)<\infty .
$$

In this case, for any function $v \in W_{2}^{1}(\omega)$, there is a $c \in \mathbb{R}$ such that

$$
\sigma(\omega, \mu)\|v-c\|_{L_{2}(\omega, \mu)} \leq\|\nabla v\|_{L_{2}(\omega)}
$$

where the constant $\sigma(\omega, \mu)>0$ does not depend on $v$ [10, Subsec. 1.4.5].

Theorem 4. Let problem (1) have a solution, and let $\mu_{k}$ be a family of measures on $\omega_{k}$, where $\omega_{k}, k=1,2, \ldots$, are pairwise disjoint Lipschitz domains in $\mathbb{R}^{n}$ such that

$$
\sup _{x \in \mathbb{R}^{n}, \rho>0} \rho^{1-n} \mu_{k}\left(B_{\rho}^{x} \cap \omega_{k}\right)<\infty
$$

and

$$
\sum_{k=1}^{\infty} \int_{\omega_{k} \backslash \Omega}|\nabla \varphi|^{2} d x<\infty
$$

Write

$$
m_{k}(\varphi)=\inf _{c \in \mathbb{R}}\|\varphi-c\|_{L_{2}\left(\omega_{k} \backslash \Omega, \mu_{k}\right)}
$$

Then

$$
\sum_{k=1}^{\infty} \sigma^{2}\left(\omega_{k}, \mu_{k}\right) m_{k}^{2}(\varphi)<\infty
$$

where $\sigma\left(\omega_{k}, \mu_{k}\right)$ stands for the coefficient in inequality (2).

To prove the Theorems $1-4$ we need a number of auxiliary results.

An inequality from the following lemma is fairly well-known [e.g., 5 p. 288, p. 398] and occurs in various forms. However, for the sake of completeness, we give a detailed proof of this inequality. 
Lemma 1 (Special Hardy inequality). Let $\psi \in C_{0}^{\infty}\left(\mathbb{R}^{n}\right)$ and $n \geq 3$. Then

$$
\int_{\mathbb{R}^{n}}|\nabla \psi|^{2} d x \geq k \int_{\mathbb{R}^{n}} \frac{|\psi|^{2}}{|x|^{2}} d x,
$$

where constant $k$ doesn't depend on $u$.

Proof. Let's pass to the polar coordinates. Hence, the integral in the righthand side takes the form

$$
\int d S \int_{0}^{\infty} \frac{|\psi|^{2}}{r^{2}} r^{n-1} d r,
$$

where first integral is taken over all angular coordinates. Let's fix angular coordinates and obtain a chain of transformations

$$
\begin{gathered}
\int_{0}^{\infty} \frac{|\psi|^{2}}{r^{2}} r^{n-1} d r=\int_{0}^{\infty}\left|\psi^{2}\right| r^{n-3} d r=\frac{1}{n-2} \int_{0}^{\infty}|\psi|^{2}\left(r^{n-2}\right)^{\prime} d r= \\
\frac{1}{n-2}\left(\left.r^{n-2}|\psi|^{2}\right|_{r=0} ^{\infty}-\int_{0}^{\infty} 2|\psi||\psi|^{\prime} r^{n-2} d r\right) .
\end{gathered}
$$

The first term in the final bracket, obviously, equals zero, as $\psi$ is a sampling function. Let's estimate the modulus of the second term, using the inequality $a b \leq \varepsilon a^{2}+\frac{1}{\varepsilon} b^{2}$, considering $a=|\psi| r^{\frac{n-3}{2}}, b=r^{\frac{n-1}{2}}\left|\psi^{\prime}\right|$.

$$
\begin{gathered}
\left.\left|\int_{0}^{\infty} 2\right| \psi|| \psi\right|^{\prime} r^{n-2} d r\left|\leq 2 \int_{0}^{\infty}\right| \psi|| \psi^{\prime} \mid r^{n-2} d r \leq \\
2\left(\varepsilon \int_{0}^{\infty}|\psi|^{2} r^{n-3} d r+\frac{1}{\varepsilon} \int_{0}^{\infty}\left|\psi^{\prime}\right|^{2} r^{n-1} d r\right) .
\end{gathered}
$$

Thus, we obtain a chain of inequalities

$$
\begin{gathered}
\int_{0}^{\infty}|\psi|^{2} r^{n-3} d r \leq \frac{2}{n-2} \int_{0}^{\infty}|\psi|\left|\psi^{\prime}\right| r^{n-2} d r \leq \\
\frac{2 \varepsilon}{n-2} \int_{0}^{\infty}|\psi|^{2} r^{n-3} d r+\frac{2}{\varepsilon(n-2)} \int_{0}^{\infty}\left|\psi^{\prime}\right|^{2} r^{n-1} d r .
\end{gathered}
$$


Consequently, by transferring of the first term to the left-hand side, we obtain following inequality

$$
\left(1-\frac{2 \varepsilon}{n-2}\right) \int_{0}^{\infty}|\psi|^{2} r^{n-3} d r \leq \frac{2}{\varepsilon(n-2)} \int_{0}^{\infty}\left|\psi^{\prime}\right|^{2} r^{n-1} d r .
$$

Given that $\left|\psi^{\prime}\right|^{2} \leq|\nabla \psi|^{2}$ and that $r^{n-1}$ represents the Jacobian of the transformation to the polar coordinates, after returning to the initial coordinates, we obtain

$$
\int_{\mathbb{R}^{n}}|\nabla \psi|^{2} d x \geq k \int_{\mathbb{R}^{n}} \frac{|\psi|^{2}}{|x|^{2}} d x
$$

Remark. Taking a sequence $\left\{\psi_{k}\right\} \in C_{0}^{\infty}\left(\mathbb{R}^{n}\right)$, which is fundamental in $L_{2}^{1}$, i.e. in seminorm

$$
\|\cdot\|_{L_{2}^{1}\left(\mathbb{R}^{n}\right)}=\left(\int_{\mathbb{R}^{n}}|\nabla \psi|^{2} d x\right)^{\frac{1}{2}},
$$

by the special Hardy inequality, we immediately obtain a fundamentality of this sequence in the metric

$$
\|\cdot\|=\left(\int_{\mathbb{R}^{n}}|\nabla \psi|^{2} d x+\int_{\mathbb{R}^{n}} \frac{|\psi|^{2}}{|x|^{2}} d x\right)^{\frac{1}{2}} .
$$

Therefore, the special Hardy inequality is also fair for $\psi \in \stackrel{\circ}{L}_{2}^{1}\left(\mathbb{R}^{n}\right)$.

Lemma 2 (General Hardy inequality). Let $u \in L_{2}^{1}\left(\mathbb{R}^{n}\right)$ and $n \geq 3$. Then there is a constant $c$ such that the following inequality is fair

$$
\int_{\mathbb{R}^{n}}|\nabla u|^{2} d x \geq k \int_{\mathbb{R}^{n}} \frac{|u-c|^{2}}{|x|^{2}} d x
$$

where constant $k$ doesn't depend on $u$.

Proof. The fact that $u$ belongs to the space $L_{2}^{1}\left(\mathbb{R}^{n}\right)$ is equivalent to the following condition

$$
\int_{\mathbb{R}^{n}}|\nabla u|^{2} d x<\infty
$$


Let's decompose the space $L_{2}^{1}\left(\mathbb{R}^{n}\right)$ into a direct product of the space ${ }_{L}^{1}\left(\mathbb{R}^{n}\right)$ and its orthogonal complement. Let $u_{0}$ be a projection of $u$ on $\stackrel{\circ}{L}_{2}^{1}\left(\mathbb{R}^{n}\right)$, and $h$ is a component from the orthogonal complement. Considering that the space ${ }^{\circ}{ }_{2}^{1}\left(\mathbb{R}^{n}\right)$ is Hilbert and separable, we find out that for any $v \in \stackrel{\circ}{L}_{2}^{1}\left(\mathbb{R}^{n}\right)$ it is true that

$$
\int_{\mathbb{R}^{n}} \nabla v \nabla h d x=0 .
$$

Hence, $\triangle h=0$ in $\mathbb{R}^{n}$. From the Parseval's identity, we obtain that

$$
\int_{\mathbb{R}^{n}}|\nabla h|^{2} d x+\int_{\mathbb{R}^{n}}\left|\nabla u_{0}\right|^{2} d x=\int_{\mathbb{R}^{n}}|\nabla u|^{2} d x .
$$

Due to the finiteness of the right-hand side, we have the finiteness of each term on the left-hand side. In particular, we obtain that

$$
\int_{\mathbb{R}^{n}}|\nabla h|^{2} d x<\infty .
$$

Recalling the ellipticity of $h$, we obtain that $h$ is constant. Then, using the special Hardy inequality with respect to $u_{0}=u-h=u-c$, we obtain the general Hardy inequality.

Lemma 3. In case of $n=2$, the general Hardy inequality takes the form

$$
\int_{\mathbb{R}^{2}}|\nabla u|^{2} d x \geq k \int_{|x| \geq 2 \delta} \frac{|u|^{2}}{|x|^{2} \ln ^{2} \frac{|x|}{\delta}} d x,
$$

for any function $u \in L_{2}^{1}\left(\mathbb{R}^{2}\right)$ and for any constant $\delta>0$, where constant $k$ doesn't depend on $u$, which is equivalent to the inequality

$$
\int_{\mathbb{R}^{2}}|\nabla u|^{2} d x \geq k \int_{\mathbb{R}^{2}} \frac{|u|^{2}}{|x|^{2} \ln ^{2}|x|} d x,
$$

for any function $u \in L_{2}^{1}\left(\mathbb{R}^{2}\right)$ such that $u=0$ almost everywhere in a neighborhood of zero, and where constant $k$ doesn't depend on $u$.

Proof. At first let's prove this proposition for a function $u \in C^{\infty}\left(\mathbb{R}^{2}\right)$. 
Let's pass to the polar coordinates. Hence, the integral in the right-hand side takes the form

$$
\int_{0}^{2 \pi} d \phi \int_{0}^{\infty} \frac{r|u|^{2}}{r^{2} \ln ^{2} r} d r
$$

Let's fix angular coordinates and obtain a chain of transformations

$$
\begin{gathered}
\int_{0}^{\infty} \frac{r|u|^{2}}{r^{2} \ln ^{2} r} d r=\int_{0}^{\infty} \frac{|u|^{2}}{r \ln ^{2} r} d r=-\int_{0}^{\infty}\left(\frac{1}{\ln r}\right)^{\prime}|u|^{2} d r= \\
-\left.\frac{1}{\ln r}|u|^{2}\right|_{r=0} ^{\infty}+\int_{0}^{\infty} \frac{1}{\ln r} 2|u||u|^{\prime} d r .
\end{gathered}
$$

The first term in the final bracket, obviously, is zero, as $u$ vanishes in a neighborhood of zero. Let's estimate the modulus of the second term, using the inequality $a b \leq \varepsilon a^{2}+\frac{1}{\varepsilon} b^{2}$, considering $a=\frac{|u|}{r^{\frac{1}{2}} \ln r}, b=r^{\frac{1}{2}}\left|u^{\prime}\right|$.

$$
\begin{gathered}
\left.\left|\int_{0}^{\infty} \frac{1}{\ln r} 2\right| u|| u\right|^{\prime} d r|=| \int_{0}^{\infty} \frac{2 r^{\frac{1}{2}}|u||u|^{\prime}}{r^{\frac{1}{2}} \ln r} d r \mid \leq 2 \int_{0}^{\infty} \frac{r^{\frac{1}{2}}|u|\left|u^{\prime}\right|}{r^{\frac{1}{2}} \ln r} d r \leq \\
2\left(\varepsilon \int_{0}^{\infty} \frac{|u|^{2}}{r \ln ^{2} r} d r+\frac{1}{\varepsilon} \int_{0}^{\infty} r\left|u^{\prime}\right|^{2} d r\right) .
\end{gathered}
$$

Thus, we obtain a chain of inequalities

$$
\int_{0}^{\infty} \frac{|u|^{2}}{r \ln ^{2} r} d r \leq 2 \varepsilon \int_{0}^{\infty} \frac{|u|^{2}}{r \ln ^{2} r} d r+\frac{2}{\varepsilon} \int_{0}^{\infty} r\left|u^{\prime}\right|^{2} d r .
$$

Consequently, by transferring of the first term to the left-hand side, we obtain the following inequality

$$
(1-2 \varepsilon) \int_{0}^{\infty} \frac{|u|^{2}}{r \ln ^{2} r} d r \leq \frac{2}{\varepsilon} \int_{0}^{\infty} r\left|u^{\prime}\right|^{2} d r .
$$

Given that $\left|u^{\prime}\right|^{2} \leq|\nabla u|^{2}$ and that $r$ represents the Jacobian of the transformation to the polar coordinates, after returning to the initial coordinates, we obtain

$$
\int_{\mathbb{R}^{2}}|\nabla u|^{2} d x \geq k \int_{\mathbb{R}^{2}} \frac{|u|^{2}}{|x|^{2} \ln ^{2}|x|} d x .
$$


Then, using the passage to the limit and the fact that $C^{\infty}$ is dense in $L_{2}^{1}[10$, p. 18], we obtain the proof of the Lemma.

Lemma 4. Let $E$ be a $(2,1)$-polar set. Then $\left.u\right|_{E}=0$ for any function $u \in W_{2, \text { loc }}^{1}\left(\mathbb{R}^{n}\right)$, i.e. $\mu u \in \stackrel{\circ}{W_{2}^{1}}\left(\mathbb{R}^{n} \backslash E\right)$ for any function $\mu \in C_{0}^{\infty}\left(\mathbb{R}^{n}\right)$.

Proof. It is known [10, p. 331, Theorem 1] that the space $\mathcal{D}(\Omega)$ is dense in $W_{2}^{1}$ if and only if $\mathbb{R}^{n} \backslash \Omega$ is a $(2,1)$-polar set. That implies the statement of the Lemma.

Lemma 5. Let $\operatorname{Cap}\left(\left(\mathbb{R}^{n} \backslash \Omega\right) \cap \bar{B}_{r_{0}}, W_{2}^{1}\left(\mathbb{R}^{n}\right)\right)>0$ for some $r_{0}$. Then

$$
\|\varphi\|_{L_{2}\left(B_{r}\right)} \leq A\|\nabla \varphi\|_{L_{2}\left(B_{r}\right)}
$$

for any $r>2 r_{0}$ and for any $\varphi \in W_{2, l o c}^{1}\left(\mathbb{R}^{n}\right)$ such that

$$
\left.\varphi\right|_{\left(\mathbb{R}^{n} \backslash \Omega\right) \cap \bar{B}_{r_{0}}}=0,
$$

where constant A doesn't depend on $\varphi$.

Proof. Let's suppose the contrary. Then for any constant $A$ there is $r>2 r_{0}$ and a function $\varphi \in W_{2, l o c}^{1}\left(\mathbb{R}^{n}\right)$ such that

$$
\left.\varphi\right|_{\left(\mathbb{R}^{n} \backslash \Omega\right) \cap \bar{B}_{r_{0}}}=0,
$$

and besides it is true that

$$
\|\varphi\|_{L_{2}\left(B_{r}\right)}>A\|\nabla \varphi\|_{L_{2}\left(B_{r}\right)}
$$

Let's choose a sequence $A_{s}=s, s=1,2, \ldots$ There is a sequence $\varphi_{s}$ such that $\left\|\varphi_{s}\right\|_{L_{2}\left(B_{r}\right)}>s\left\|\nabla \varphi_{s}\right\|_{L_{2}\left(B_{r}\right)}$. Denote

$$
\psi_{s}=\frac{\varphi_{s}}{\left\|\varphi_{s}\right\|_{L_{2}\left(B_{r}\right)}} .
$$

It is obvious that $\left\|\psi_{s}\right\|_{L_{2}\left(B_{r}\right)}=1$, while

$$
\left\|\nabla \psi_{s}\right\|_{L_{2}\left(B_{r}\right)} \rightarrow 0 \text { as } s \rightarrow \infty .
$$


Consequently, $\left\|k-\psi_{s}\right\|_{W_{2}^{1}\left(B_{r}\right)}$ tends to zero as $s \rightarrow \infty$ for some constant $k$. Thus, taking the function $\left(k-\psi_{s}\right) \eta$, where $\eta \in C_{0}^{\infty}\left(B_{2 r_{0}}\right), \eta \equiv 1$ in a neighborhood of $B_{r_{0}}$, we obtain that

$\operatorname{Cap}\left(\left(\mathbb{R}^{n} \backslash \Omega\right) \cap \bar{B}_{r_{0}}, W_{2}^{1}\left(\mathbb{R}^{n}\right)\right) \leq \int_{\mathbb{R}^{n}}\left|\nabla\left(\left(k-\psi_{s}\right) \eta\right)\right|^{2} d x \leq$ const $\left\|k-\psi_{s}\right\|_{W_{2}^{1}\left(B_{r}\right)}$.

Taking the limit as $s \rightarrow \infty$, it follows that $\operatorname{Cap}\left(\left(\mathbb{R}^{n} \backslash \Omega\right) \cap \bar{B}_{r_{0}}, W_{2}^{1}\left(\mathbb{R}^{n}\right)\right)=0$. This contradiction proves the Lemma.

Proof of the Theorem 1. $\left\{r_{i}\right\}_{i=1}^{n}$ and $\left\{\rho_{i}\right\}_{i=1}^{n}$ are infinitely increasing sequences of real numbers. Let $r_{i}<\rho_{i}$ for all $i$, and

$$
\operatorname{cap}_{\varphi-c}\left(\left(\mathbb{R}^{n} \backslash \Omega\right) \cap \bar{B}_{r_{i}}, B_{\rho_{i}}\right)<\operatorname{cap}_{\varphi-c}\left(\mathbb{R}^{n} \backslash \Omega\right)+\frac{1}{2^{i}}, i=1,2, \ldots
$$

It is obvious [11] that capacity $\operatorname{cap}_{\varphi-c}\left(\left(\mathbb{R}^{n} \backslash \Omega\right) \cap \bar{B}_{r_{i}}, B_{\rho_{i}}\right)$ is achieved by the function $v_{i} \in \stackrel{\circ}{W}_{2}^{1}\left(B_{\rho_{i}}\right)$ such that

$$
\left\{\begin{array}{l}
\triangle v_{i}=0 \quad B_{\rho_{i}} \backslash\left(\left(\mathbb{R}^{n} \backslash \Omega\right) \cap \bar{B}_{r_{i}}\right) \\
\left.v_{i}\right|_{\left(\mathbb{R}^{n} \backslash \Omega\right) \cap \bar{B}_{r_{i}}}=\varphi-c,
\end{array}\right.
$$

where the last equality means that $\left(v_{i}-(\varphi-c)\right) \mu \in \stackrel{\circ}{W_{2}^{1}}\left(B_{\rho_{i}} \backslash\left(\left(\mathbb{R}^{n} \backslash \Omega\right) \cap \bar{B}_{r_{i}}\right)\right)$ for any $\mu \in C_{0}^{\infty}\left(B_{\rho_{i}}\right)$. Along with the problem (5), let's consider another problem:

$$
\left\{\begin{array}{l}
L u_{i}=0 \text { on } B_{\rho_{i}} \backslash\left(\left(\mathbb{R}^{n} \backslash \Omega\right) \cap \bar{B}_{r_{i}}\right) \\
\left.u_{i}\right|_{\left(\mathbb{R}^{n} \backslash \Omega\right) \cap \bar{B}_{r_{i}}}=\varphi-c
\end{array}\right.
$$

where $u_{i} \in \stackrel{\circ}{W}{ }_{2}^{1}\left(B_{\rho_{i}}\right)$.

The following statement takes place: let function $u_{i}$ be a solution of the problem (6), and function $v_{i}$ is a solution of the problem (5). Then

$$
\int_{B_{\rho_{i}}}\left|\nabla v_{i}\right|^{2} d x \leq \int_{B_{\rho_{i}}}\left|\nabla u_{i}\right|^{2} d x \leq c \int_{B_{\rho_{i}}}\left|\nabla v_{i}\right|^{2} d x
$$

where $c$ is a non-negative constant, which doesn't depend on $u_{i}$ and $v_{i}$. Let's prove this fact. The left-hand inequality, obviously, follows from the definition 
of capacity. Let's prove the right-hand inequality. Given that the function $u_{i}$ is a solution of the problem (6), it is true that

$$
\int_{B_{\rho_{i}}} \sum_{l, m=1}^{n} a_{l m}(x) \frac{\partial u_{i}}{\partial x_{m}} \frac{\partial \psi}{\partial x_{l}} d x=0
$$

for any function $\psi \in \stackrel{\circ}{W}_{2}^{1}(\Omega)$. In particular, taking $\psi=u-v$, we obtain

$$
\int_{B_{\rho_{i}}} \sum_{l, m=1}^{n} a_{l m}(x) \frac{\partial u_{i}}{\partial x_{m}} \frac{\partial u_{i}}{\partial x_{l}} d x-\int_{B_{\rho_{i}}} \sum_{l, m=1}^{n} a_{l m}(x) \frac{\partial u_{i}}{\partial x_{m}} \frac{\partial v_{i}}{\partial x_{l}} d x=0,
$$

from where the following estimates are obtained

$$
\begin{gathered}
\gamma \int_{B_{\rho_{i}}}\left|\nabla u_{i}\right|^{2} d x \leq \int_{B_{\rho_{i}}} \sum_{l, m=1}^{n} a_{l m}(x) \frac{\partial u_{i}}{\partial x_{m}} \frac{\partial u_{i}}{\partial x_{l}} d x=\int_{B_{\rho_{i}}} \sum_{l, m=1}^{n} a_{l m}(x) \frac{\partial u_{i}}{\partial x_{m}} \frac{\partial v_{i}}{\partial x_{l}} d x \leq \\
\text { const }\left(\int_{B_{\rho_{i}}}\left|\nabla u_{i}\right|^{2} d x\right)^{\frac{1}{2}}\left(\int_{\Omega}\left|\nabla v_{i}\right|^{2} d x\right)^{\frac{1}{2}},
\end{gathered}
$$

that prove the right-hand inequality in (7).

It is obvious that

$$
\operatorname{cap}_{\varphi-c}\left(\left(\mathbb{R}^{n} \backslash \Omega\right) \cap \bar{B}_{r_{i}}\right) \leq \int_{B_{\rho_{i}}}\left|\nabla v_{i}\right|^{2} d x<\operatorname{cap}_{\varphi-c}\left(\mathbb{R}^{n} \backslash \Omega\right)+\frac{1}{2^{i}} .
$$

At the same time, from the inequality (7), it follows that

$$
\int_{B_{\rho_{i}}}\left|\nabla u_{i}\right|^{2} d x<c\left(\operatorname{cap}_{\varphi-c}\left(\mathbb{R}^{n} \backslash \Omega\right)+\frac{1}{2^{i}}\right) .
$$

If $\operatorname{Cap}\left(\mathbb{R}^{n} \backslash \Omega, W_{2}^{1}\left(\mathbb{R}^{n}\right)\right)=0$ then the set $\mathbb{R}^{n} \backslash \Omega$ is $(2,1)$-polar [10, p. 331], what means, according to the Lemma 4 , that the function $1-\varphi$ is zero on $\mathbb{R}^{n} \backslash \Omega$. Thus, taking the unit function, we obtain the required solution of the problem (1).

Now let $\operatorname{Cap}\left(\left(\mathbb{R}^{n} \backslash \Omega\right) \cap \bar{B}_{r_{i}}, W_{2}^{1}\left(\mathbb{R}^{n}\right)\right)>0$ for some $r_{i}$. Then, from the Lemma 5, we obtain that the sequence $\left\{u_{i}\right\}_{i=1}^{n}$ is bounded both in $L_{2}\left(B_{r}\right)$ and $W_{2}^{1}\left(B_{r}\right)$ for any $r$. Indeed, for sufficiently large $i, j$ it is true that

$$
u_{i}-u_{j} \in \stackrel{\circ}{W}_{2, l o c}^{1}\left(\mathbb{R}^{n} \backslash\left(\left(\mathbb{R}^{n} \backslash \Omega\right) \cap \bar{B}_{r}\right)\right), \quad i, j>i_{0} .
$$


Thus, fixing $j$, we have

$$
\left\|u_{i}\right\|_{L_{2}^{1}}^{2}+\left\|u_{i}\right\|_{W_{2}^{1}(K)}^{2} \leq \alpha\left(\left\|u_{j}\right\|_{W_{2}^{1}(K)}^{2}+\operatorname{cap}_{\varphi-c}\left(\mathbb{R}^{n} \backslash \Omega\right)+\frac{1}{2^{i}}+\frac{1}{2^{j}}\right)
$$

for any compacta $K \subset \mathbb{R}^{n}$, where constant $\alpha>0$ doesn't depend on $u_{i}$.

Due to the compactness of the embedding $W_{2}^{1}\left(B_{r}\right)$ in $L_{2}\left(B_{r}\right)$, we can choose a subsequence of the sequence $\left\{u_{i}\right\}_{i=1}^{n}$ which is fundamental in $L_{2}\left(B_{r}\right)$. In order not to overload the indexes, we denote this subsequence also as $\left\{u_{i}\right\}_{i=1}^{n}$. Let's take a function $\eta \in C_{0}^{\infty}\left(B_{r}\right)$ such that $\eta \equiv 1$ in an open neighborhood of the set $\bar{B}_{r / 2}$. Due to the fact that $u_{i}$ satisfies (6), for the difference $u_{i}-u_{j}$ we obtain that

$$
\int_{B_{r}} \sum_{l, m=1}^{n} a_{l m}(x) \frac{\partial\left(u_{i}-u_{j}\right)}{\partial x_{m}} \frac{\partial \psi}{\partial x_{l}} d x=0
$$

where $\psi=\eta^{2}\left(u_{i}-u_{j}\right)$. In other words,

$$
\begin{gathered}
\int_{B_{r}} \sum_{l, m=1}^{n} a_{l m}(x) \frac{\partial\left(u_{i}-u_{j}\right)}{\partial x_{m}} \frac{\partial \eta^{2}}{\partial x_{l}}\left(u_{i}-u_{j}\right) d x+ \\
\int_{B_{r}} \sum_{l, m=1}^{n} a_{l m}(x) \eta^{2} \frac{\partial\left(u_{i}-u_{j}\right)}{\partial x_{m}} \frac{\partial\left(u_{i}-u_{j}\right)}{\partial x_{l}} d x=0 .
\end{gathered}
$$

Let's rewrite the last relation in the form

$$
\gamma \int_{B_{r}} \eta^{2}\left|\nabla\left(u_{i}-u_{j}\right)\right|^{2} d x \leq-2 \int_{B_{r}} \sum_{k, l=1}^{n} a_{k l}(x) \frac{\partial\left(u_{i}-u_{j}\right)}{\partial x_{l}} \frac{\partial \eta}{\partial x_{k}} \eta\left(u_{i}-u_{j}\right) d x,
$$

whence, in view of the inequality $a b \leq \frac{1}{2} a^{2}+\frac{1}{2} b^{2}$, we obtain that

$$
\int_{B_{r}} \eta^{2}\left|\nabla\left(u_{i}-u_{j}\right)\right|^{2} d x \leq c_{1} \int_{B_{r}}\left|\nabla\left(u_{i}-u_{j}\right)\right|^{2} \eta^{2} d x+c_{2} \int_{B_{r}}|\nabla \eta|^{2}\left(u_{i}-u_{j}\right)^{2} d x,
$$

where $c_{1}, c_{2}$ are non-negative constants, which don't depend on $u_{i}$. Thus, we have

$$
\int_{B_{r / 2}}\left|\nabla\left(u_{i}-u_{j}\right)\right|^{2} d x \leq \beta \int_{B_{r}}\left(u_{i}-u_{j}\right)^{2} d x
$$

where constant $\beta>0$ doesn't depend on $u_{i}$. Last inequality proves that the sequence $\left\{u_{i}\right\}_{i=1}^{n}$ is fundamental in $W_{2}^{1}\left(B_{r / 2}\right)$ for any $r>0$. Therefore, there 
is a function $u \in W_{2, l o c}^{1}\left(\mathbb{R}^{n}\right)$ such that for any $r>0$ the sequence $\left\{u_{i}\right\}_{i=1}^{n}$ tends to $u$ in $W_{2}^{1}\left(B_{r}\right)$. It is obvious that the function $u$ is the desired solution of the problem (1).

Proof of the Theorem 2. Let's suppose that the function $u$ is a solution of the problem (1). Let's extend $u$ on $\mathbb{R}^{n} \backslash \Omega$ with value $\varphi$. Let $n \geq 3$, then there is a constant $c \in \mathbb{R}$ such that for the function $u$ the general Hardy inequality takes place. Denote

$$
\nu_{R}=\eta\left(\frac{|x|}{R}\right)(u-c),
$$

where $\eta \in C_{0}^{\infty}\left(B_{2}\right)$ and $\eta \equiv 1$ in an open neighborhood of the set $\bar{B}_{1}$. Hence, we obtain

$$
\left.\nu_{R}\right|_{\left(\mathbb{R}^{n} \backslash \Omega\right) \cap \bar{B}_{R}}=\varphi-c .
$$

The Dirichlet integral for the function $\nu_{R}$ can be estimated

$$
\begin{gathered}
\int_{B_{2 R}}\left|\nabla\left(\eta\left(\frac{|x|}{R}\right)(u-c)\right)\right|^{2} d x \leq \\
2\left(\int_{B_{2 R}}\left|\nabla \eta\left(\frac{|x|}{R}\right)(u-c)\right|^{2} d x+\int_{B_{2 R}}\left|\eta\left(\frac{|x|}{R}\right) \nabla(u-c)\right|^{2} d x\right\rfloor .
\end{gathered}
$$

Let's notice that

$$
\left|\nabla \eta\left(\frac{|x|}{R}\right)\right| \leq \frac{p}{R} \text { and } \frac{1}{R^{2}} \leq \frac{4}{|x|^{2}} \text { for } x \in B_{2 R},
$$

where $p>0$ is a constant. Then, considering the Hardy inequality, we obtain

$$
\begin{gathered}
\int_{B_{2 R}}\left|\nabla \eta\left(\frac{|x|}{R}\right)(u-c)\right|^{2} d x \leq \frac{p^{2}}{R^{2}} \int_{B_{2 R} \backslash B_{R}}|u-c|^{2} d x \leq \\
4 p^{2} \int_{B_{2 R} \backslash B_{R}} \frac{|u-c|^{2}}{|x|^{2}} d x \leq \frac{4 p^{2}}{k} \int_{B_{2 R}}|\nabla u|^{2} d x .
\end{gathered}
$$

Thus,

$$
\operatorname{cap}_{\varphi-c}\left(\left(\mathbb{R}^{n} \backslash \Omega\right) \cap \bar{B}_{R}\right) \leq \int_{B_{2 R}}\left|\nabla \nu_{R}\right|^{2} d x \leq \gamma \int_{B_{2 R}}|\nabla u|^{2} d x<\infty,
$$


where $\gamma>0$ is a constant, which doesn't depend on $\nu_{R}$. Proceeding to the limit as $R \rightarrow \infty$, we obtain

$$
\operatorname{cap}_{\varphi-c}\left(\mathbb{R}^{n} \backslash \Omega\right) \leq \gamma \int_{\mathbb{R}^{n}}|\nabla u|^{2} d x<\infty,
$$

that proves the Theorem 2 for $n \geq 3$.

In case of $n=2$, denoting

$$
\nu_{R}=\eta\left(\frac{\ln \frac{|x|}{R}}{\ln R}\right) u,
$$

where $\eta \in C_{0}^{\infty}\left(\mathbb{R}^{2}\right), \eta=0$ in a neighborhood of zero and $\eta \equiv 1$ in a open neighborhood of $B_{1}$, we obtain

$$
\left.\nu_{R}\right|_{\left(\mathbb{R}^{n} \backslash \Omega\right) \cap \bar{B}_{R^{2}}}=\varphi .
$$

The Dirichlet integral for the function $\nu_{R}$ can be estimated

$$
\begin{gathered}
\int_{B_{2 R^{2}}}\left|\nabla\left(\eta\left(\frac{\ln \frac{|x|}{R}}{\ln R}\right) u\right)\right|^{2} d x \leq \\
2\left(\int_{B_{2 R^{2}}}\left|\nabla \eta\left(\frac{\ln \frac{|x|}{R}}{\ln R}\right) u\right|^{2} d x+\int_{B_{2 R^{2}}}\left|\eta\left(\frac{\ln \frac{|x|}{R}}{\ln R}\right) \nabla u\right|^{2} d x \mid .\right.
\end{gathered}
$$

Let's notice that

$$
\left|\nabla \eta\left(\frac{\ln \frac{|x|}{R}}{\ln R}\right)\right| \leq \frac{2 q}{|x| \ln R^{2}} \text { and } \frac{1}{\ln ^{2} R^{2}} \leq \frac{m}{\ln ^{2}|x|} \text { for } x \in B_{2 R^{2}},
$$

where $q, m>0$ are some constants. Then, considering the Hardy inequality, we obtain

$$
\begin{gathered}
\int_{B_{2 R^{2}}}\left|\nabla \eta\left(\frac{\ln \frac{|x|}{R}}{\ln R}\right) u\right|^{2} d x \leq \int_{B_{2 R^{2} \backslash B_{R^{2}}} \frac{4 q^{2}}{|x|^{2} \ln ^{2} R^{2}}|u|^{2} d x \leq} \\
4 q^{2} m \int_{B_{2 R^{2} \backslash B_{R^{2}}}} \frac{|u|^{2}}{|x|^{2} \ln ^{2}|x|} d x \leq \frac{4 q^{2} m}{k} \int_{B_{2 R^{2}}}|\nabla u|^{2} d x .
\end{gathered}
$$


Thus,

$$
\operatorname{cap}_{\varphi}\left(\left(\mathbb{R}^{2} \backslash \Omega\right) \cap \bar{B}_{R^{2}}\right) \leq \int_{B_{2 R^{2}}}\left|\nabla \nu_{R}\right|^{2} d x \leq \gamma \int_{B_{2 R^{2}}}|\nabla u|^{2} d x<\infty,
$$

where $\gamma>0$ is a constant, which doesn't depend on $\nu_{R}$. Proceeding to the limit as $R \rightarrow \infty$, we obtain

$$
\operatorname{cap}_{\varphi}\left(\mathbb{R}^{2} \backslash \Omega\right) \leq \gamma \int_{\mathbb{R}^{2}}|\nabla u|^{2} d x<\infty .
$$

Thus, the Theorem 2 is completely proved.

Proof of the Theorem 3. Let cap $\varphi_{\varphi-c}\left(\mathbb{R}^{n} \backslash \Omega\right)<\infty$. Then, by the Theorem 2 , there is a function $u$, which is a solution of the problem (1). Let $n \geq 3$. Let's consider a shearing function $\eta_{k} \in C_{0}^{\infty}\left(\mathbb{R}^{n}\right)$ such that $\eta_{k}(x)=1$ on $B_{2^{k+1}} \backslash B_{2^{k-1}}$ and $\operatorname{supp} \eta_{k}(x) \subset B_{2^{k+2}} \backslash B_{2^{k-2}}, k=1,2, \ldots$, which is constructed as follows. Let $\eta(x)$ is a monotone non-decreasing function from $C^{\infty}\left(\mathbb{R}^{n}\right)$, which is equal to zero on the interval $\left[-\infty, \frac{1}{4}\right]$ and is equal to one on the interval $\left[\frac{3}{4},+\infty\right]$. Further, we denote by $\eta_{k}(x)$ the following function

$$
\eta_{k}(x)= \begin{cases}\eta\left(\frac{|x|-r_{k-2}}{r_{k-1}-r_{k-2}}\right), & \text { if } x \in \bar{B}_{r_{k-1}} \backslash B_{r_{k-2}} \\ 1, & \text { if } x \in \bar{B}_{r_{k+1}} \backslash B_{r_{k-1}} \\ \eta\left(\frac{r_{k+2}-|x|}{r_{k+2}-r_{k+1}}\right), & \text { if } x \in \bar{B}_{r_{k+2}} \backslash B_{r_{k+1}} .\end{cases}
$$

We have the estimate

$$
\left|\nabla \eta_{k}(x)\right|^{2} \leq \frac{c}{|x|^{2}}
$$

where $c$ doesn't depend on $k$. Then, considering the Hardy inequality, we obtain a chain of inequalities

$$
\begin{gathered}
\operatorname{cap}_{\varphi-c}\left(\left(\bar{B}_{r_{k+1}} \backslash B_{r_{k-1}}\right) \cap\left(\mathbb{R}^{n} \backslash \Omega\right), B_{r_{k+2}} \backslash \bar{B}_{r_{k-2}}\right) \leq \\
\int_{B_{r_{k+2}} \backslash \bar{B}_{r_{k-2}}}\left|\nabla\left(\eta_{k}(x) u(x)\right)\right|^{2} d x \leq 2 \int_{B_{r_{k+2}} \backslash \bar{B}_{r_{k-2}}}\left|\nabla \eta_{k}(x) u(x)\right|^{2} d x+ \\
2 \int_{B_{r_{k+2}} \backslash \bar{B}_{r_{k-2}}}\left|\eta_{k}(x) \nabla u(x)\right|^{2} d x \leq 2 c \int_{B_{r_{k+2}} \backslash_{\bar{B}_{r_{k-2}}}} \frac{|u(x)|^{2}}{|x|^{2}} d x+
\end{gathered}
$$




$$
b_{1} \int_{B_{r_{k+2}} \backslash \bar{B}_{r_{k-2}}}|\nabla u(x)|^{2} d x
$$

where $b_{1}$ is a positive constant, which doesn't depend on $u$. Thus,

$$
\begin{gathered}
\sum_{k=1}^{\infty} \operatorname{cap}_{\varphi-c}\left(\left(\bar{B}_{r_{k+1}} \backslash B_{r_{k-1}}\right) \cap\left(\mathbb{R}^{n} \backslash \Omega\right), B_{r_{k+2}} \backslash \bar{B}_{r_{k-2}}\right) \leq \\
\sum_{k=1}^{\infty} 2 \int_{B_{r_{k+2}} \backslash \bar{B}_{r_{k-2}}} \frac{|u(x)|^{2}}{|x|^{2}} d x+\sum_{k=1}^{\infty} b_{1} \int_{B_{r_{k+2}} \backslash \bar{B}_{r_{k-2}}}|\nabla u(x)|^{2} d x .
\end{gathered}
$$

Due to the fact that each point $x \in \mathbb{R}^{n}$ belongs to no more than three areas $B_{r_{k+2}} \backslash \bar{B}_{r_{k-2}}$, we obtain

$$
\begin{aligned}
& \sum_{k=1}^{\infty} 2 \int_{B_{r_{k+2}} \backslash \bar{B}_{r_{k-2}}} \frac{|u(x)|^{2}}{|x|^{2}} d x+\sum_{k=1}^{\infty} b_{1} \int_{B_{r_{k+2}} \backslash \bar{B}_{r_{k-2}}}|\nabla u(x)|^{2} d x \leq \\
& b_{2} \int_{\mathbb{R}^{n}} \frac{|u(x)|^{2}}{|x|^{2}} d x+b_{3} \int_{\mathbb{R}^{n}}|\nabla u(x)|^{2} d x \leq b_{4} \int_{\mathbb{R}^{n}}|\nabla u(x)|^{2} d x<\infty,
\end{aligned}
$$

where $b_{2}, b_{3}, b_{4}$ are positive constants, which don't depend on $u$.

Now let $n=2$. Denote

$$
\eta_{k}(x)= \begin{cases}\eta\left(\frac{\ln \frac{|x|}{r_{k-2}}}{\left.\ln \frac{r_{k-1}}{r_{k-2}}\right),}\right. & \text { if } x \in \bar{B}_{r_{k-1}} \backslash B_{r_{k-2}} \\ 1, & \text { if } x \in \bar{B}_{r_{k+1}} \backslash B_{r_{k-1}} \\ \eta\left(\frac{\ln \frac{r_{k+2}}{|x|}}{\left.\ln \frac{r_{k+2}}{r_{k+1}}\right),}\right. & \text { if } x \in \bar{B}_{r_{k+2}} \backslash B_{r_{k+1}},\end{cases}
$$

where $\eta(x)$ is a monotone non-decreasing function from $C^{\infty}\left(\mathbb{R}^{n}\right)$, which is equal to zero on the interval $\left[-\infty, \frac{1}{4}\right]$ and is equal to one on the interval $\left[\frac{3}{4},+\infty\right]$. We have the estimate

$$
\left|\nabla \eta_{k}(x)\right|^{2} \leq \frac{c}{|x|^{2} \ln ^{2}|x|},
$$

where $c$ doesn't depend on $k$. Then, considering the Hardy inequality, we obtain a chain of inequalities

$$
\operatorname{cap}_{\varphi-c}\left(\left(\bar{B}_{r_{k+1}} \backslash B_{r_{k-1}}\right) \cap\left(\mathbb{R}^{n} \backslash \Omega\right), B_{r_{k+2}} \backslash \bar{B}_{r_{k-2}}\right) \leq
$$




$$
\begin{gathered}
\int_{B_{r_{k+2}} \backslash \bar{B}_{r_{k-2}}}\left|\nabla\left(\eta_{k}(x) u(x)\right)\right|^{2} d x \leq 2 \int_{B_{r_{k+2}} \backslash \bar{B}_{r_{k-2}}}\left|\nabla \eta_{k}(x) u(x)\right|^{2} d x+ \\
2 \int_{B_{r_{k+2}} \backslash \bar{B}_{r_{k-2}}}\left|\eta_{k}(x) \nabla u(x)\right|^{2} d x \leq 2 c \int_{B_{r_{k+2}} \backslash \bar{B}_{r_{k-2}}} \frac{|u(x)|^{2}}{|x|^{2} \ln ^{2}|x|} d x+ \\
b_{1} \int_{B_{r_{k+2}} \backslash \bar{B}_{r_{k-2}}}|\nabla u(x)|^{2} d x,
\end{gathered}
$$

where $b_{1}$ is a positive constant, which doesn't depend on $u$. Thus,

$$
\begin{gathered}
\sum_{k=1}^{\infty} \operatorname{cap}_{\varphi-c}\left(\left(\bar{B}_{r_{k+1}} \backslash B_{r_{k-1}}\right) \cap\left(\mathbb{R}^{n} \backslash \Omega\right), B_{r_{k+2}} \backslash \bar{B}_{r_{k-2}}\right) \leq \\
\sum_{k=1}^{\infty} 2 \int_{B_{r_{k+2}} \backslash \bar{B}_{r_{k-2}}} \frac{|u(x)|^{2}}{|x|^{2} \ln ^{2}|x|} d x+\sum_{k=1}^{\infty} b_{1} \int_{B_{r_{k+2}} \backslash \bar{B}_{r_{k-2}}}|\nabla u(x)|^{2} d x .
\end{gathered}
$$

Due to the fact that each point $x \in \mathbb{R}^{n}$ belongs to no more than three areas $B_{r_{k+2}} \backslash \bar{B}_{r_{k-2}}$, we obtain

$$
\begin{aligned}
& \sum_{k=1}^{\infty} 2 \int_{B_{r_{k+2}} \backslash \bar{B}_{r_{k-2}}} \frac{|u(x)|^{2}}{|x|^{2} \ln ^{2}|x|} d x+\sum_{k=1}^{\infty} b_{1} \int_{B_{r_{k+2}} \backslash \bar{B}_{r_{k-2}}}|\nabla u(x)|^{2} d x \leq \\
& b_{2} \int_{\mathbb{R}^{n}} \frac{|u(x)|^{2}}{|x|^{2} \ln ^{2}|x|} d x+b_{3} \int_{\mathbb{R}^{n}}|\nabla u(x)|^{2} d x \leq b_{4} \int_{\mathbb{R}^{n}}|\nabla u(x)|^{2} d x<\infty,
\end{aligned}
$$

where $b_{2}, b_{3}, b_{4}$ are positive constants, which don't depend on $u$.

The converse. Let

$$
\sum_{k=1}^{\infty} \operatorname{cap}_{\varphi-c}\left(\left(\bar{B}_{r_{k+1}} \backslash B_{r_{k-1}}\right) \cap\left(\mathbb{R}^{n} \backslash \Omega\right), B_{r_{k+2}} \backslash \bar{B}_{r_{k-2}}\right)<\infty
$$

and $n \geq 3$. Let's consider a shearing function

$$
\widetilde{\psi}_{k}(x)= \begin{cases}\eta\left(\frac{|x|-r_{k-1}}{r_{k}-r_{k-1}}\right), & \text { if }|x| \leq r_{k} \\ \eta\left(\frac{r_{k+1}-|x|}{r_{k+1}-r_{k}}\right), & \text { if }|x| \geq r_{k} .\end{cases}
$$


Denote

$$
\psi_{k}(x)=\frac{\widetilde{\psi}_{k}(x)}{\sum_{i=0}^{\infty} \widetilde{\psi}_{i}(x)}
$$

Obviously,

$$
\sum_{k=1}^{\infty} \psi_{k}(x)=1
$$

From the condition on the capacity, we have functions $u_{k}(x)$, which implement the capacity and equal to $\varphi-c$ on $B_{r_{k+1}} \backslash B_{r_{k-1}}$ and with supports from $B_{r_{k+2}} \backslash$ $B_{r_{k-2}}$. Let's notice that

$$
\sum_{k=N_{1}}^{N_{2}} u_{k}(x) \psi_{k}(x)=\varphi-c
$$

if $x$ is from a neighborhood of the set $\left(\bar{B}_{r_{N_{2}-1}} \backslash B_{r_{N_{1}+1}}\right) \cap\left(\mathbb{R}^{n} \backslash \Omega\right)$. Then we obtain

$$
\begin{gathered}
\left|\nabla \sum_{k=N_{1}}^{N_{2}} u_{k}(x) \psi_{k}(x)\right|^{2}=\left|\sum_{k=N_{1}}^{N_{2}} \nabla u_{k}(x) \psi_{k}(x)+\sum_{k=N_{1}}^{N_{2}} u_{k}(x) \nabla \psi_{k}(x)\right|^{2} \leq \\
2\left|\sum_{k=N_{1}}^{N_{2}} \nabla u_{k}(x) \psi_{k}(x)\right|^{2}+2\left|\sum_{k=N_{1}}^{N_{2}} u_{k}(x) \nabla \psi_{k}(x)\right|^{2} .
\end{gathered}
$$

Since for each $x \in \mathbb{R}^{n}$ there are no more than three natural numbers $k \in$ $\left\{N_{1}, \ldots, N_{2}\right\}$ such that $\psi_{k}(x) \neq 0$, then we obtain

$$
\left|\sum_{k=N_{1}}^{N_{2}} \nabla u_{k}(x) \psi_{k}(x)\right|^{2} \leq 9 \sum_{k=N_{1}}^{N_{2}}\left|\nabla u_{k}(x)\right|^{2}\left|\psi_{k}(x)\right|^{2} .
$$

Similarly,

$$
\left|\sum_{k=N_{1}}^{N_{2}} u_{k}(x) \nabla \psi_{k}(x)\right|^{2} \leq 9 \sum_{k=N_{1}}^{N_{2}}\left|u_{k}(x)\right|^{2}\left|\nabla \psi_{k}(x)\right|^{2}
$$

As a result, we obtain

$$
\left|\nabla \sum_{k=N_{1}}^{N_{2}} u_{k}(x) \psi_{k}(x)\right|^{2} \leq 18 \sum_{k=N_{1}}^{N_{2}}\left|\nabla u_{k}(x)\right|^{2}\left|\psi_{k}(x)\right|^{2}+18 \sum_{k=N_{1}}^{N_{2}}\left|\nabla u_{k}(x)\right|^{2}\left|\psi_{k}(x)\right|^{2} .
$$


Therefore,

$$
\begin{gathered}
\int_{\mathbb{R}^{n}} \mid \nabla\left(\sum_{k=N_{1}}^{N_{2}} u_{k}(x) \psi_{k}(x)||^{2} d x \leq 18\left(\sum_{k=N_{1} B_{r_{k+2}} \backslash B_{r_{k-2}}}^{N_{2}}\left|\nabla u_{k}(x) \psi_{k}(x)\right|^{2} d x+\right.\right. \\
\sum_{k=N_{1}}^{N_{2}} \int_{B_{r_{k+2}} \backslash B_{r_{k-2}}}\left|u_{k}(x) \nabla \psi_{k}(x)\right|^{2} d x \mid \cdot
\end{gathered}
$$

The first term in the last expression can be estimated as follows

$$
\sum_{k=N_{1}}^{N_{2}} \int_{B_{r_{k+2}} \backslash B_{r_{k-2}}}\left|\nabla u_{k}(x) \psi_{k}(x)\right|^{2} d x \leq \sum_{k=N_{1}}^{N_{2}} \int_{B_{r_{k+2}} \backslash B_{r_{k-2}}}\left|\nabla u_{k}(x)\right|^{2} d x,
$$

since $\left|\psi_{k}(x)\right| \leq 1$. Using the fact that

$$
\left|\nabla \psi_{k}(x)\right| \leq \frac{r_{k+1}-r_{k}}{2^{k}}
$$

and Friedrichs' inequality, we estimate the second term as follows

$$
\begin{gathered}
\sum_{k=N_{1}}^{N_{2}} \int_{B_{r_{k+2}} \backslash B_{r_{k-2}}}\left|u_{k}(x) \nabla \psi_{k}(x)\right|^{2} d x \leq \sum_{k=N_{1}}^{N_{2}} \frac{\left(r_{k+1}-r_{k}\right)^{2}}{4^{k}} \int_{B_{r_{k+2}} \backslash B_{r_{k-2}}}\left|u_{k}(x)\right|^{2} d x \leq \\
c_{1} \sum_{k=N_{1}}^{N_{2}} \int_{B_{r_{k+2}} \backslash B_{r_{k-2}}}\left|\nabla u_{k}(x)\right|^{2} d x,
\end{gathered}
$$

where $c_{1}$ is a positive constant, which doesn't depend on $u_{k}$ and $\psi_{k}$. We obtain a chain of inequalities

$$
\begin{gathered}
\operatorname{cap}_{\varphi-c}\left(\left(\bar{B}_{r_{N_{2}}} \backslash B_{r_{N_{1}}}\right) \cap\left(\mathbb{R}^{n} \backslash \Omega\right)\right) \leq \int_{\mathbb{R}^{n}}\left|\nabla\left(\sum_{k=N_{1}}^{N_{2}} u_{k}(x) \psi_{k}(x)\right)\right|^{2} d x \leq \\
c_{2} \sum_{k=N_{1}}^{N_{2}} \int_{B_{r_{k+2}} \backslash B_{r_{k-2}}}\left|\nabla u_{k}(x)\right|^{2} d x= \\
\sum_{k=N_{1}}^{N_{2}} \operatorname{cap}_{\varphi-c}\left(\left(\bar{B}_{r_{k+1}} \backslash B_{r_{k-1}}\right) \cap\left(\mathbb{R}^{n} \backslash \Omega\right), B_{r_{k+2}} \backslash \bar{B}_{r_{k-2}}\right),
\end{gathered}
$$


where $c_{2}$ is a positive constant, which doesn't depend on $u_{k}$ and $\psi_{k}$. As $N_{2}$ tending to infinity, we obtain

$$
\begin{gathered}
\operatorname{cap}_{\varphi-c}\left(\left(\mathbb{R}^{n} \backslash \Omega\right) \backslash B_{r_{N_{1}}}\right) \leq \\
\sum_{k=N_{1}}^{\infty} \operatorname{cap}_{\varphi-c}\left(\left(\bar{B}_{r_{k+1}} \backslash B_{r_{k-1}}\right) \cap\left(\mathbb{R}^{n} \backslash \Omega\right), B_{r_{k+2}} \backslash \bar{B}_{r_{k-2}}\right)<\infty,
\end{gathered}
$$

what implies that

$$
\operatorname{cap}_{\varphi-c}\left(\mathbb{R}^{n} \backslash \Omega\right)<\infty .
$$

Now let $n=2$. Then let's consider a shearing function

$$
\widetilde{\psi}_{k}(x)= \begin{cases}\eta\left(\frac{\ln \frac{|x|}{r_{k-1}}}{\ln \frac{r_{k}}{r_{k-1}}}\right), & \text { if }|x| \leq r_{k} \\ \eta\left(\frac{\left.\ln \frac{r_{k+1}}{\ln \frac{r_{k+1}}{r_{k}}}\right),}{}\right) & \text { if }|x| \geq r_{k},\end{cases}
$$

and let

$$
\psi_{k}(x)=\frac{\widetilde{\psi}_{k}(x)}{\sum_{i=0}^{\infty} \widetilde{\psi}_{i}(x)} .
$$

Obviously,

$$
\sum_{k=1}^{\infty} \psi_{k}(x)=1
$$

From the condition on the capacity, we have functions $u_{k}(x)$, which implement the capacity and equal to $\varphi-c$ on $B_{r_{k+1}} \backslash B_{r_{k-1}}$ and with supports from $B_{r_{k+2}} \backslash$ $B_{r_{k-2}}$. Let's notice that

$$
\sum_{k=N_{1}}^{N_{2}} u_{k}(x) \psi_{k}(x)=\varphi-c
$$

if $x$ is from a neighborhood of the set $\left(\bar{B}_{r_{N_{2}-1}} \backslash B_{r_{N_{1}+1}}\right) \cap\left(\mathbb{R}^{n} \backslash \Omega\right)$. It is easy to see that the functions $\psi_{k}$ again satisfy the following relations

$$
\int_{\mathbb{R}^{n}} \mid \nabla\left(\left.\sum_{k=N_{1}}^{N_{2}} u_{k}(x) \psi_{k}(x)\right|^{2} d x \leq 18\left(\sum_{k=N_{1}}^{N_{2}} \int_{B_{r_{k+2}} \backslash B_{r_{k-2}}}\left|\nabla u_{k}(x) \psi_{k}(x)\right|^{2} d x+\right.\right.
$$




$$
\sum_{k=N_{1}}^{N_{2}} \int_{B_{r_{k+2}} \backslash B_{r_{k-2}}}\left|u_{k}(x) \nabla \psi_{k}(x)\right|^{2} d x \mid
$$

The first term in the last expression can be estimated as follows

$$
\sum_{k=N_{1}}^{N_{2}} \int_{B_{r_{k+2}} \backslash B_{r_{k-2}}}\left|\nabla u_{k}(x) \psi_{k}(x)\right|^{2} d x \leq \sum_{k=N_{1} B_{r_{k+2}}}^{N_{2}} \int_{B_{r_{k-2}}}\left|\nabla u_{k}(x)\right|^{2} d x
$$

since $\left|\psi_{k}(x)\right| \leq 1$. Then, using the fact that $\left|\nabla \psi_{k}(x)\right| \leq \frac{\text { const }}{|x| \ln |x|}$ and the Hardy inequality, we estimate the second term as follows

$$
\begin{aligned}
& \sum_{k=N_{1}}^{N_{2}} \int_{B_{r_{k+2}} \backslash B_{r_{k-2}}}\left|u_{k}(x) \nabla \psi_{k}(x)\right|^{2} d x \leq \sum_{k=N_{1}}^{N_{2}} \int_{B_{r_{k+2}} \backslash B_{r_{k-2}}} \frac{\left|u_{k}(x)\right|^{2}}{|x|^{2} \ln ^{2}|x|} d x \leq \\
& c_{1} \sum_{k=N_{1}}^{N_{2}} \int_{B_{r_{k+2}} \backslash B_{r_{k-2}}}\left|\nabla u_{k}(x)\right|^{2} d x
\end{aligned}
$$

where $c_{1}$ is a positive constant, which doesn't depend on $u_{k}$ and $\psi_{k}$. We obtain a chain of inequalities

$$
\begin{aligned}
& \operatorname{cap}_{\varphi-c}\left(\left(\bar{B}_{r_{N_{2}}} \backslash B_{r_{N_{1}}}\right) \cap\left(\mathbb{R}^{n} \backslash \Omega\right)\right) \leq \int_{\mathbb{R}^{n}}\left|\nabla\left(\sum_{k=N_{1}}^{N_{2}} u_{k}(x) \psi_{k}(x)\right)\right|^{2} d x \leq \\
& c_{2} \sum_{k=N_{1}}^{N_{2}} \int_{B_{r_{k+2}} \backslash B_{r_{k-2}}}\left|\nabla u_{k}(x)\right|^{2} d x= \\
& \sum_{k=N_{1}}^{N_{2}} \operatorname{cap}_{\varphi-c}\left(\left(\bar{B}_{r_{k+1}} \backslash B_{r_{k-1}}\right) \cap\left(\mathbb{R}^{n} \backslash \Omega\right), B_{r_{k+2}} \backslash \bar{B}_{r_{k-2}}\right),
\end{aligned}
$$

where $c_{2}$ is a positive constant, which doesn't depend on $u_{k}$ and $\psi_{k}$. As $N_{2}$ tending to infinity, we obtain

$$
\begin{gathered}
\operatorname{cap}_{\varphi-c}\left(\left(\mathbb{R}^{n} \backslash \Omega\right) \backslash B_{r_{N_{1}}}\right) \leq \\
\sum_{k=N_{1}}^{\infty} \operatorname{cap}_{\varphi-c}\left(\left(\bar{B}_{r_{k+1}} \backslash B_{r_{k-1}}\right) \cap\left(\mathbb{R}^{n} \backslash \Omega\right), B_{r_{k+2}} \backslash \bar{B}_{r_{k-2}}\right)<\infty
\end{gathered}
$$


what implies that

$$
\operatorname{cap}_{\varphi-c}\left(\mathbb{R}^{n} \backslash \Omega\right)<\infty
$$

Proof of the Theorem 4. Let $u$ is a solution of the problem (1). Let's extend $u$ on $\mathbb{R}^{n} \backslash \Omega$ with value $\varphi$. Then, by the inequality (2), we obtain that

$$
\sigma\left(\omega_{k}, \mu_{k}\right)\left\|u-c_{k}\right\|_{L_{2}\left(\omega_{k}, \mu_{k}\right)} \leq\|\nabla u\|_{L_{2}\left(\omega_{k}\right)},
$$

what implies that

$$
\sigma^{2}\left(\omega_{k}, \mu_{k}\right)\left\|\varphi-c_{k}\right\|_{L_{2}\left(\omega_{k} \backslash \Omega, \mu_{k}\right)}^{2}=\sigma^{2}\left(\omega_{k}, \mu_{k}\right)\left\|u-c_{k}\right\|_{L_{2}\left(\omega_{k} \backslash \Omega, \mu_{k}\right)}^{2} \leq\|\nabla u\|_{L_{2}\left(\omega_{k}\right)}^{2} .
$$

Summing this relation, we obtain

$$
\begin{gathered}
\sum_{k=1}^{\infty} \sigma^{2}\left(\omega_{k}, \mu_{k}\right)\left\|\varphi-c_{k}\right\|_{L_{2}\left(\omega_{k} \backslash \Omega, \mu_{k}\right)}^{2} \leq \sum_{k=1}^{\infty}\|\nabla u\|_{L_{2}\left(\omega_{k}\right)}^{2}= \\
\sum_{k=1}^{\infty} \int_{\omega_{k} \cap \Omega}|\nabla u|^{2} d x+\sum_{k=1}^{\infty} \int_{\omega_{k} \backslash \Omega}|\nabla u|^{2} d x .
\end{gathered}
$$

Let's notice that

$$
\sum_{k=1}^{\infty} \int_{\omega_{k} \cap \Omega}|\nabla u|^{2} d x \leq \int_{\Omega}|\nabla u|^{2} d x<\infty,
$$

and

$$
\sum_{k=1}^{\infty} \int_{\omega_{k} \backslash \Omega}|\nabla u|^{2} d x=\sum_{k=1}^{\infty} \int_{\omega_{k} \backslash \Omega}|\nabla \varphi|^{2} d x<\infty .
$$

Thus, we have

$$
\sum_{k=1}^{\infty} \sigma^{2}\left(\omega_{k}, \mu_{k}\right)\left\|\varphi-c_{k}\right\|_{L_{2}\left(\omega_{k} \backslash \Omega, \mu_{k}\right)}^{2}<\infty
$$

which immediately implies (4). The Theorem is completely proved. 


\section{Acknowledgments}

The author expresses his gratitude to Professor A.A. Kon'kov for setting the problem and for the interest shown in the process of its solution.

\section{References}

[1] A.L. Beklaryan, Existence theorems for elliptic equations in unbounded domains, Proceedings of III International conference "Optimization and applications" (OPTIMA-2012), Portugal (2012), 47-50.

[2] A.L. Beklaryan, On the Existence of Solutions of the First Boundary Value Problem for Elliptic Equations on Unbounded Domains, Russian Journal of Mathematical Physics, 19, No. 4 (2012), 508-511, DOI: 10.1134/S1061920812040115.

[3] L. Bers, F. John, M. Schechter, Partial differential equations, American Mathematical Soc., USA (1964).

[4] I.M. Gel'fand, G.E. Shilov, Generalized functions. Vol. I: Properties and operations, MA: Academic Press, Boston (1964).

[5] G.H. Hardy, J.E. Littlewood, G. Polya, Inequalities, Cambridge University Press, New York-London-Tokyo (1952).

[6] A.N. Kolmogorov, S.V. Fomin, Elements of the Theory of Functions and Functional Analysis, Dover Publications, USA (1999).

[7] V.A. Kondrat'ev, The Solvability of the First Boundary Value Problem for Strongly Elliptic Equations, Tr. Mosk. Mat. Obs., 16 (1967), 209-292 [in Russian].

[8] A.A. Kon'kov, On the Dimension of the Solution Space of Elliptic Systems in Unbounded Domains, Russ. Acad. Sci. Sb. Math., 80, No. 2 (1995), 411-434, DOI: 10.1070/SM1995v080n02ABEH003531.

[9] O.A. Ladyzhenskaya, N.N. Ural'tseva, Linear and Quasilinear Elliptic Equations, Academic Press, New York-London (1968).

[10] V.G. Maz'ya, Sobolev Spaces, Springer-Verlag, Berlin-New York (1985).

[11] S.G. Mihlin, Linear partial differential equations, Vysshaya shkola, Russia (1977) [in Russian]. 
[12] V.S. Vladimirov, Equations of mathematical physics, Mir, Moscow (1984). 